\title{
Post-Training Isoflurane Anesthesia Affects Memory for Object Recognition but Not for Two-Way Active Avoidance Task
}

\author{
Patricia Jurado-Berbel, David Costa-Miserachs", Meritxell Torras-Garcia, Margalida Coll-Andreu, \\ Laura Aldavert-Vera and Isabel Portell-Cortés
}

\author{
Departament de Psicobiologia i de Metodologia de les Ciències de la Salut, Institut de Neurociències, Edifici B, \\ Universitat Autònoma de Barcelona, 08193-Bellaterra, Spain
}

\begin{abstract}
Certain experimental procedures in learning and memory research require that the animal be anesthetized soon after the acquisition session. Volatile anesthetics, such as isoflurane, are good candidates for this purpose because they have both a rapid onset of effect and a quick awakening of the animal when anesthesia is discontinued. However, memory consolidation might be affected by a mixed effect of the anesthetic agent itself and the administration procedures. In the present experiment, we examined, in male Wistar rats, the effect of 15 or 60 min post-training isoflurane anesthesia on 24 $\mathrm{h}$ object recognition memory, a task depending on perirhinal cortex and hippocampus (Experiment I), and on $24 \mathrm{~h}$ or 20 days retention of a two-way active avoidance task, mainly depending on striatum and amygdala (Experiment II). The results showed that the global procedure of anesthesia with isoflurane impaired object recognition memory, but did not affect retention of two-way active avoidance, neither when tested $24 \mathrm{~h}$ nor 20 days after the acquisition session. We concluded that the differential effect of the treatment in these two tasks might be accounted for by an interaction of the specific features of the task and the stress produced by the administration procedure of the anesthetic agent.
\end{abstract}

Keywords: Learning, memory, post-training anesthesia, volatile anesthetics, object recognition, two-way active avoidance.

\section{INTRODUCTION}

The study of neural mechanisms involved in memory consolidation requires posttraining treatments to avoid the effects on other processes such as the encoding of information or attention that could confound the results. Some of these experimental strategies may need to anesthetize the animal, when painful or seizuring treatments (for example, excitotoxic lesions) have to be applied in a post-training basis. For this kind of experiments, volatile anesthetics, such as isoflurane, are especially recommended because of the rapid onset and offset of their hypnotic effect. However, both volatile anesthetics and their administration procedures could affect memory consolidation.

Concerning volatile anesthetics, there are several pieces of evidence that they can induce both anterograde and retrograde effects on memory, although there are still many open questions. With regard to anterograde effects, isoflurane administration can affect memory for tasks learned several days or even months later. Amongst other factors, the effects seem to depend on the age of the animal, the kind of learning task, the amount of training, and the delay between anesthesia and training [see, for example, references 1-5].

With regard to retrograde effects, mixed results have also been found after posttraining anesthesia with volatile anesthetics. For example, some studies have found no effect on retention of fear conditioning [4], inhibitory avoidance,

*Address correspondence to this author at the Departament de Psicobiologia i de Metodologia de les Ciències de la Salut, Institut de Neurociències, Edifici B, Universitat Autònoma de Barcelona, 08193-Bellaterra, Spain; Tel: (34) 935.812.543; Fax: (34) 935.812.001; E-mail: david.costa@uab.cat active avoidance [6], or a well learned spatial task [7], but other studies have reported an improvement in memory for active avoidance [8], and spatial tasks [9]. Also with a spatial task, Culley et al. [10] reported that post-training isoflurane improved performance in young rats but impaired it in aged rats. Furthermore, in an early study of post-training etherinduced anesthesia in mice, an impairment of retention in a one-trial inhibitory avoidance conditioning task was found [11]. Overall, it is not clear what the effect of volatile anesthetics on retrograde memory is.

Beside the direct influence of the specific anesthetic agent used, the procedures required for administering it may also exert some effects on learning and memory. To administer volatile anesthetic agents, the common practice is to place the animal in a relatively small box (induction chamber), and then administer the gas. After initial induction, anesthesia must be continued in order to administer the posttraining treatment (for example, for intracranial infusion of a neurotoxin). In rodent brain research experiments, anesthesia maintenance is generally provided through a face mask. Although anesthetic induction is very quick, the placement of the animals in the box may cause some stress. Moreover, when recovering from anesthesia, the animals might also suffer some distress. It is important to take this issue into account, since it is well known that endogenous and exogenous increases in stress related hormones, such as epinephrine and glucocorticoids, can have both facilitatory and disruptive effects on memory, depending on the dose [1216].

Due to these possible effects of both volatile anesthetics and the administration procedure, when a post-training anesthesia is needed in a memory experiment, it would be nece- 
ssary to assess the effect, on memory, of the global procedure of anesthesia (including placement in the induction box and maintenance in a stereotaxic mask), rather than that of the anesthetic agent alone. From the existing literature we cannot predict the way memory would be affected by the global anesthesia procedure with a volatile agent. Furthermore, as in the case of other treatments, the effect of the global anesthesia procedure might depend on the memory task, the retention delay, and the duration of the treatment, among other factors.

According to those considerations, the main aims of the present study was to determine whether the global anesthesia procedure with isoflurane (i.e, the effects of the anesthetic agent summated to those of the administration procedures) affects memory when this treatment takes place immediately after a single learning session. We studied this considering the three following conditions:

\section{The Memory Task}

In experiment 1 we used a novel object recognition memory task, which appears to be related to the perirhinal cortex and hippocampus [17-20]. In Experiment II, animals were trained in a massed session of a two-way active avoidance conditioning, a task involving both classical and instrumental conditioning that is considered to be related mainly to striatum and the amygdaloid complex [21-25].

\section{The Retention Delay}

The effect of the global anesthetic procedure with isoflurane on long-term memory was evaluated $24 \mathrm{~h}$ after acquisition for novel object recognition task, and $24 \mathrm{~h}$ or 20 days after the training session for active avoidance task. For this latter task we measured two different delays of retention because it has been shown that the effect of some treatments on this task might be observed on long-term memory, with a 20-day delay between acquisition and memory tests [26].

\section{The Duration of Anesthesia}

For the two tasks, we tested two durations of posttraining anesthesia, 15 and 60 minutes.

\section{MATERIALS AND METHODS}

\section{Experiment I}

\section{Subjects}

We used thirty-one naïve male Wistar rats, obtained from our laboratory breeding stock, with a mean age of 97.45 days $(\mathrm{SD}=2.54)$ and a mean weight of $424.65 \mathrm{~g}(\mathrm{SD}=40.87)$ at the beginning of the experiment. All rats were housed singly in $22 \times 22 \times 14-\mathrm{cm}$ plastic-bottomed cages with sawdust bedding, kept under conditions of controlled temperature $\left(20-22^{\circ} \mathrm{C}\right)$ and humidity $(40-70 \%)$, and maintained on a 12 hour light-dark cycle (lights on at 8:00 a.m.). Experiments were performed during the first half of the light phase of the cycle. Rat-chow pellets (Panlab S.L, A04) and water were provided ad libitum. All procedures were performed in compliance with the European Community Council directive for care and use of laboratory animals (86/609/EEC) and with the related directive of the Autonomous Government of
Catalonia (DOGC 2073 10/7/1995). The experiment was approved by the Ethics Committee for Animal and Human Experimentation of the Autonomous University of Barcelona (permit number 3185).

\section{Apparatus of Object Recognition and Neophobia Test}

The apparatus consisted of an open box $(65 \mathrm{~cm}$ width $\mathrm{x}$ $65 \mathrm{~cm}$ length $\times 35 \mathrm{~cm}$ height) made of conglomerate covered with brown melamine. The illumination on the floor of the box was 50-60 lux. Objects were constructed from Lego that varied in shape, color, and size. Since the objects were made of the same material, they could not be distinguished by olfactory cues. The objects were weighted so that the animals could not move them around the arena. They were not known to have any ethological significance for the rats and they had never been paired with a reinforcer. Three different objects were used, two for the object recognition task and one for the previous neophobia test. The objects for the recognition task were available in duplicate copies. All behavioral sessions were recorded with a video camera (Canon MVX10i) mounted above the experimental apparatus. Tapes were analyzed off-line by an observer who was unaware of the treatment condition.

\section{Post-Training Treatment}

Animals were anesthetized initially with 5\% isoflurane (Forane ${ }^{\circledR}$, Abbot Laboratories, SA, Madrid, Spain) in oxygen $(0.81 / \mathrm{min})$ in a Plexiglas chamber $(20 \times 13 \times 13 \mathrm{~cm})$ for 3 min. Thereafter the animals were placed on a small electrical mat to keep their body temperature, and maintained with $2.5 \%-3.5 \%$ isoflurane in oxygen $(0.41 / \mathrm{min})$ for 15 (Iso15 group) or 60 minutes (Iso-60 group) with a face mask, a condition that may be suitable when certain posttraining procedures (intracranial infusion of a neurotoxin, for example) are required. Anesthesia depth was continuously monitored through visual inspection, as well as through tailpinching whenever necessary. To absorb anesthetic vapors from the work area and protect the experimenters from exposure, an scavenging system (Fluovac System, Harvard Apparatus, with a Fluosorber canister inserted) was used. Control rats were returned to their home cage after the termination of the acquisition session (Ctrl group).

\section{Handling and Habituation}

Animals were handled for approximately 5 minutes on two consecutive days after being housed singly. Two days after the last handling sessions, the rats were habituated to the experimental apparatus with 6 sessions of $15 \mathrm{~min}$ of exploration in the absence of objects on three consecutive days ( 2 sessions per day, 2 hr-delay).

\section{Neophobia Test}

The day after the last habituation session, a neophobia test was conducted [27]. An unfamiliar object was exposed in the center of the open box. The animals were placed in the box facing away from the object and allowed to explore the object for $5 \mathrm{~min}$. The latency of the first exploration of the object and the total time exploring the object were recorded. Throughout the experiment, exploration of an object was defined as directing the nose to the object at a distance $\leq 2$ $\mathrm{cm}$ or touching it with the nose. Turning around or sitting on the object was not considered exploratory behavior. 


\section{Object Recognition Task}

Object recognition training began $24 \mathrm{~h}$ after the neophobia test. In the training session, two identical objects were placed near the two corners at either end of one side of the cage. The rat was placed in the experimental apparatus, facing the center of the opposite wall and was allowed to explore the two objects for $15 \mathrm{~min}$. To avoid the presence of olfactory cues, the apparatus and objects were thoroughly cleaned with a vinegar solution $(20 \%)$ and dried before the first rat and after each animal. The time spent in exploring each object was recorded. An exclusion criterion of at least $20 \mathrm{~s}$ of total exploration in the training trial was established.

Immediately after the acquisition session, animals were anesthetized with isoflurane for either 15 (Iso-15 group, $\mathrm{n}=10$ ) or 60 (Iso-60 group, $\mathrm{n}=10$ ) minutes, and then returned to their home cage. Control rats were returned to their home cage after the termination of the acquisition session (Ctrl group, $\mathrm{n}=11$ ). The rats were randomly assigned to these treatment groups.

Retention was tested $24 \mathrm{~h}$ after the training session. In the retention test, one copy of the familiar object and a new object were placed in the same location as the stimuli used during the training trial. The specific objects used as either familiar or novel, as well as their locations, were used in a balanced manner to reduce potential biases due to preferences for particular location or object. The rats were placed in the open box for $5 \mathrm{~min}$. The time spent exploring each object was recorded, for the first minute and for the whole retention test. To analyze cognitive performance, a discrimination index was calculated ([time exploring the novel object - time exploring the familiar object / total time spent on both objects] x 100). This kind of ratio makes it possible to adjust for any differences in total exploration time [28]. This task is based on the natural tendency of rats to explore novelty, so that an index significantly higher than zero (i.e., animals exploring the novel object more than the familiar one) is considered a good recall of the familiar object, whereas an index close to zero (i.e., animals exploring both objects similarly) is considered a lack of recall [27].

\section{Statistics}

The statistical computer package program SPSS 14.0 (Chicago, IL) was used to analyze the data. One-way analysis of variance (ANOVA) was applied considering Group (Ctrl, Iso-15, Iso-60) as the independent variable, and the first exploration latency and total exploration time in the neophobia test or the discrimination index in the acquisition (left $v s$. right) and retention (novel $v s$. familiar) sessions as the dependent variables. For both training and retention sessions, the total exploration time of the two objects was also considered a dependent variable. Differences between groups were determined by a unilateral Dunnett post-hoc analysis. One-sample t-tests were used to determine whether the discrimination index was different from zero (chance level). Statistical significance was set at the level of $P \leq$ 0.05 .

\section{Experiment 2}

\section{Subjects}

We used seventy naïve male Wistar rats, obtained from our laboratory breeding stock, with a mean age of 98.90 days $(\mathrm{SD}=7.62)$ and a mean weight of $435.43 \mathrm{~g}(\mathrm{SD}=41.12)$ at the beginning of the experiment. The animals were housed and maintained under the same conditions described for Experiment I. All procedures were performed in compliance with the European Community Council directive for care and use of laboratory animals (86/609/EEC) and with the related directive of the Autonomous Government of Catalonia (DOGC 2073 10/7/1995). The experiment was approved by the Ethics Committee for Animal and Human Experimentation of the Autonomous University of Barcelona (permit number 2779).

\section{Apparatus}

Two-way active avoidance conditioning was conducted in a $50 \times 24 \times 23 \mathrm{~cm}$ automated shuttle box (Letica LI-916, Panlab,SL, Barcelona, Spain) constructed of Plexiglas. Compartment floors (without any physical separation between them) were independently electrifiable and constructed of stainless-steel bars. The shuttle box was enclosed in a sound-attenuating box ventilated by an extractor fan. The box was illuminated by a fluorescent light located inside the sound-attenuating box. The conditioned stimulus (CS) was a $60-\mathrm{dB}$ and $1-\mathrm{KHz}$ tone of $3 \mathrm{~s}$ duration. The unconditioned stimulus (US) was a $0.5-\mathrm{mA}$ electrical footshock, presented immediately after the end of the CS, for a maximum of $15 \mathrm{~s}$. The trials followed a variable interval schedule of $60 \mathrm{~s}( \pm$ 10s).

\section{Post-Training Treatment}

The post-training treatment was the same as in experiment I.

\section{Two-Way Avoidance Task Procedure}

The animals were handled for approximately 5 minutes on the two consecutive days after being housed singly. On the third day, animals were trained in a two-way active avoidance task session (30 trials). Prior to the conditioning session, the rats were allowed to ambulate freely in the shuttle box for $10 \mathrm{~min}$ to become familiarized with the learning context [29]. The conditioned stimulus (CS) was a $60-\mathrm{dB}$ and $1-\mathrm{KHz}$ tone of a $3-\mathrm{sec}$ duration. The unconditioned stimulus (US) was presented on termination of the CS and consisted of a $0.5-\mathrm{mA}$ scrambled electrical footshock. During this session, rats could avoid the shock by crossing to the adjacent compartment when the CS was on (avoidance response). The shuttle box was connected to a computer that controlled the training schedule and scored avoidance responses (considered to be the level of performance of the task), escape responses, and the number of intertrial crossings and crossings during the free ambulation period (considered an index of locomotor activity).

Immediately after the acquisition session, animals were anesthetized with isoflurane for 15 (Iso-15 groups) or $60 \mathrm{~min}$ (Iso-60 groups). Control rats were returned to their home 
cage after the termination of the acquisition session (Ctrl groups). The rats were randomly assigned to these treatment groups.

To test the retention of the learned response, rats received one additional avoidance session that was identical to the acquisition session (10 min of free ambulation in the shuttle box followed by 30 trials) $24 \mathrm{~h}$ or 21 days after the acquisition session. The $10 \mathrm{~min}$ free ambulation period was included to reduce poor performance at the beginning of the retention session caused by the warm-up effect [30]. The rats were randomly assigned to test retention at $24 \mathrm{~h}$ or 21 days.

\section{Statistics}

The statistical computer package program SPSS 14.0 (Chicago, IL) was used to analyze the data. The main analyses were carried out by means of analyses of variance considering the independent variables to be qualitative (posttraining treatment: Ctrl, Iso-15 and Iso-60; retention interval: $24 \mathrm{hr}$ and 21 days), and the dependent variable to be quantitative (repeated measures, performance in each session, acquisition and retention: avoidances or crossings). Considering that the retention session involves a large number of trials with an increasing additional learning component as the session goes on, we also analyzed performance during the first 10 trials of the retention session, that have a greater retention component, and compared this with the performance in the last 10 trials of the acquisition session [12].

Eight rats displayed abnormal behavior in the acquisition session (less than 2 avoidance responses) and were excluded from the analyses. The final sample was composed of sixtytwo rats distributed into the 24-hr retention groups (Iso-15, $\mathrm{n}=10$; Iso-60, $\mathrm{n}=10 ; \mathrm{Ctrl}, \mathrm{n}=11)$ and the 21-day retention groups (Iso-15, $\mathrm{n}=10 ;$ Iso- $60=10 ; \mathrm{Ctrl}, \mathrm{n}=11$ ). Due to problems with the software used to collect shuttle box data, it was not possible to record the number of intertrial crossings and crossings during the free ambulation period for one rat (control group tested 21 days after the acquisition session). Statistical significance was set at $P \leq 0.05$.

\section{RESULTS}

\section{Experiment 1}

\section{Neophobia Test}

There were no significant differences between groups in total exploration time, $\mathrm{F}(2,30)=0.99, \mathrm{p}=\mathrm{NS}$, and latency of the first approach to the object, $\mathrm{F}(2,30)=0.25, \mathrm{p}=\mathrm{NS}$, during the neophobia test, prior to any pharmacological treatment.

\section{Training Session}

No animal met the exclusion criteria $(<20 \quad \mathrm{~s}$ of exploration during the training session). ANOVA revealed no differences between groups, $F(2,30)=0.02, p=N S$, in the mean of the total exploration time of the two identical objects in the training session. Moreover, the analysis revealed no differences between groups in the discrimination index, showing that all groups exhibited comparable amounts of time exploring each of the two (left and right) identical objects, $\mathrm{F}(2,30)=2.20, \mathrm{p}=\mathrm{NS}$.

\section{Retention Session}

Fig. (1) shows the discrimination index in the retention session. This index was only significantly different from zero for the control group, both when considering the first minute, $\mathrm{t}(10)=6.01, \mathrm{p}<.001$, or the whole of the retention session, $\mathrm{t}(10)=3.64, \mathrm{p}=.005$. That is, control animals showed a preference for exploring the novel object, indicating memory of the familiar object in the 24-hr retention test. In contrast, no exploration preferences for the novel object in the isoflurane-treated rats was found, in both the Iso-15 and Iso-60 group [Iso-15: first minute $\mathrm{t}(10)=$ $0.44, \mathrm{p}=\mathrm{NS}$, whole session $\mathrm{t}(10)=0.86, \mathrm{p}=\mathrm{NS}$; Iso-60: first minute $\mathrm{t}(10)=1.11, \mathrm{p}=\mathrm{NS}$, whole session $\mathrm{t}(10)=0.31, \mathrm{p}=$ NS]. Moreover, differences between groups were observed when the first minute, $\mathrm{F}(2,30)=3.26, \mathrm{p}=0.05$, but not the whole, $\mathrm{F}(2,30)=2.20, \mathrm{p}=\mathrm{NS}$, of the retention session was considered. Post-hoc contrast analyses revealed that 15 minutes of exposure to isoflurane significantly decreased the

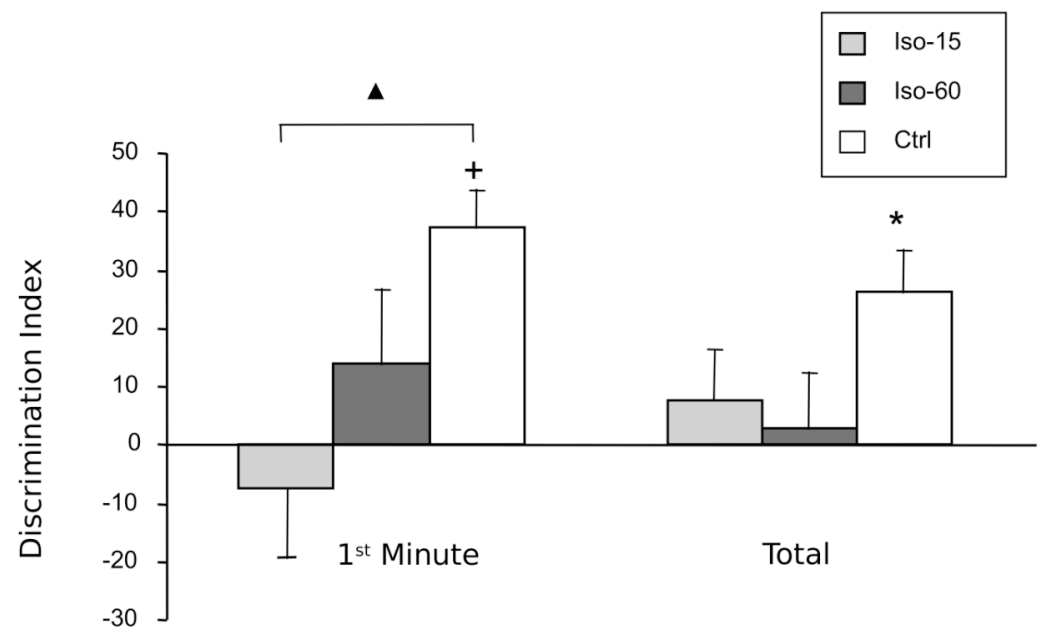

Fig. (1). Mean value ( \pm S.E.M.) of the discrimination index in the object recognition task. Only control animals showed a preference for exploring the novel object, indicating memory of the familiar object in the 24-hr retention test, both when considering the first minute $(+, p<$ $0.001)$ or the whole of the retention session $(*, p<0.005) .15$ minutes of exposition to isoflurane significantly decreased the discrimination index concerning the first minute of the retention session compared with controls $(\boldsymbol{\Delta}=0.03)$. 
discrimination index concerning the first minute of the retention session compared with controls, bilateral t-Dunnett, $\mathrm{p}=0.03$.

The statistical analyses showed no differences between groups in the total exploration time of the two objects in the retention test, $\mathrm{F}(2,30)=0.68, \mathrm{p}=\mathrm{NS}$. These findings indicate that post-training anesthesia with isoflurane did not influence the total amount of time spent exploring the two objects in the retention test.

\section{Experiment 2}

Fig. (2) depicts the mean number of avoidances in the acquisition and retention sessions. As seen in this figure, the number of avoidance responses increased in all the groups in the retention session compared to the acquisition session, the level of avoidances in each of these sessions being similar in all groups. The analyses of variance showed that there were differences between sessions, $\mathrm{F}(1,56)=104.47, \mathrm{p}<0.001$, but not between groups, $\mathrm{F}(2,56)=0.02, \mathrm{p}=\mathrm{NS}$, or retention delays, $\mathrm{F}(1,56)=2.98, \mathrm{p}=\mathrm{NS}$. The interaction between the two main factors was also non significant, F (2, $56)=0.8, p=$ NS. Similar results were obtained when we analyzed the first 10 trials of the retention session, as well as the evolution from the last 10 trials of the acquisition session. Again, the analyses of variance showed that only the session factor, $F(1,56)=27.66, \mathrm{p}<0.001$, but not the group, $\mathrm{F}(2,56)=0.16, \mathrm{p}=\mathrm{NS}$, the retention delay, $\mathrm{F}(1,56)$ $=0.8, \mathrm{p}=\mathrm{NS}$, or the interaction effects, $\mathrm{F}(2,56)=0.25, \mathrm{p}=$ NS, was statistically significant.

The examination of the locomotor activity revealed similar results. The mean number of crossings made by the subjects during the free pre-session ambulation and the intertrial periods significantly decreased from the acquisition to the retention session [pre-session, $F(1,55)=41.92, p<$ 0.001 ; inter-trial, $\mathrm{F}(1,55)=32.39, \mathrm{p}<0.001]$. Neither the group [pre-session, $\mathrm{F}(2,55)=0.03, \mathrm{p}=\mathrm{NS}$; inter-trial, $\mathrm{F}(2$, $55)=0.34, \mathrm{p}=\mathrm{NS}$ ], the retention delay [pre-session, $\mathrm{F}(1$, $55)=3.40, \mathrm{p}=\mathrm{NS}$; inter-trial, $\mathrm{F}(1,55)=1.05, \mathrm{p}=\mathrm{NS}]$ nor the interaction [pre-session, $\mathrm{F}(2,55)=0.67, \mathrm{p}=\mathrm{NS}$; inter- trial, $\mathrm{F}(2,55)=0.10, \mathrm{p}=\mathrm{NS}$ ] effects were statistically significant.

\section{DISCUSSION}

The main purpose of the present work was to study whether the global anesthesia procedure with isoflurane can affect memory consolidation when applied immediately after training, and whether the effect might depend on the task, the duration of the anesthetic treatment, and the interval of retention (in the case of two-way active avoidance conditioning). Our results support the view that this treatment can affect the memory for object recognition.

We found that both 15 and $60 \mathrm{~min}$ of immediate posttraining anesthesia with isoflurane impaired 24-h memory for the novel object recognition task (experiment I). Specifically, in the 24-h retention session the animals in the control group explored the novel object more than the familiar one, whereas rats in the isoflurane groups explored both objects similarly. As explained in the method section, a higher exploration of the novel than the familiar object is interpreted to be a good recall of the task, whereas similar exploration of both objects takes place when rats do not remember the task [27]. Thus, the control group, but not the isoflurane groups, remembered the task. In addition, $15 \mathrm{~min}$ of exposure to isoflurane significantly decreased the discrimination index in the first minute, compared to the control group. Regarding two-way active avoidance we found that 15 or $60 \mathrm{~min}$ of immediate post-training anesthesia with isoflurane did not affect $24 \mathrm{~h}$ or 21 day retention, neither when considering the whole session nor the first ten trials of the retention session (experiment II). So, the lack of any effect of the global anesthesia procedure on this task does not seem to be due to the influence of additional learning during the retention session.

Some studies have found that isoflurane can induce hyperlocomotion [31], but our results do not seem attributable to changes in motor activity. Although a direct measure of locomotion was not recorded in Experiment I, the fact that differences were found in the discrimination index, but not in

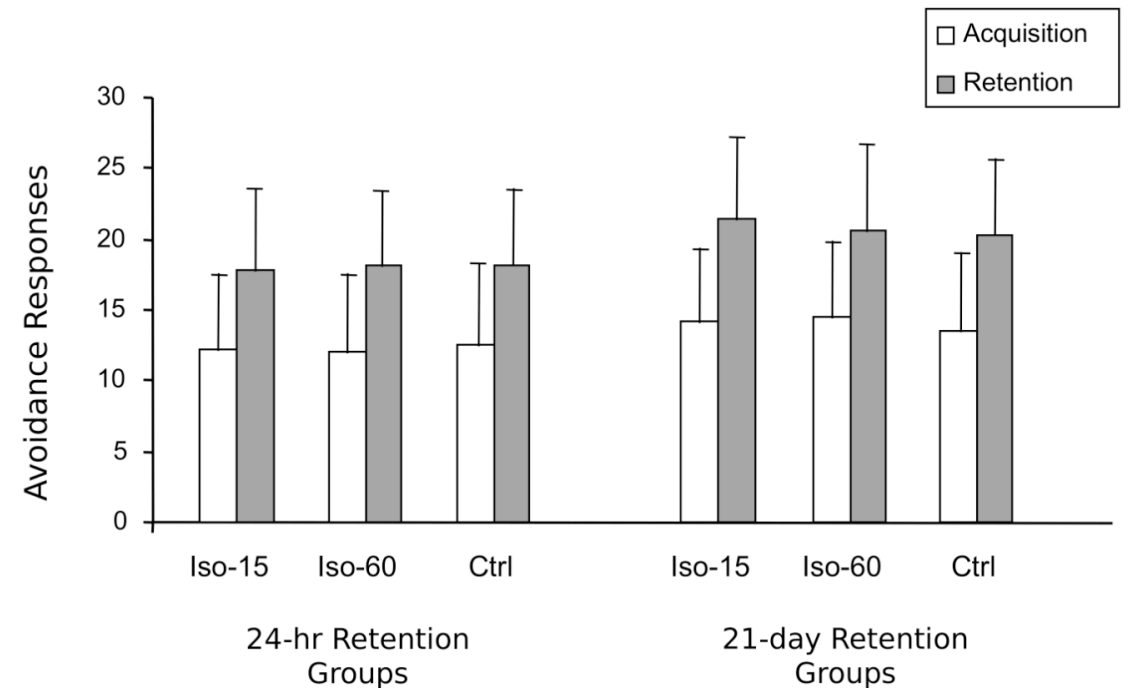

Fig. (2). Mean number of avoidances ( \pm S.E.M.) in the acquisition and retention sessions of two-way active avoidance task. No statistically significant differences were observed between groups. 
the total amount of object exploration during the retention test, suggests that the deleterious effect on retention can hardly be attributable to any proactive effect of isoflurane on locomotion. Moreover, no differences in the locomotor measures recorded were found in Experiment II. Therefore, the lack of effects in the latter experiment can not be explained by any increase in motor activity that could have masked an impairing effect of the anesthetic treatment in the retention test.

The differences in recall found in Experiment I do not seem attributable to differences in basal emotional reactivity, since the performance of all the groups during the neophobia test was similar. Our results cannot be explained, either, by a poor level of learning in the anesthetized groups, because the level of object exploration in the acquisition session was similar in all groups and seemed to be enough to allow memory formation (as evidenced by the good recall shown by control rats). A possible influence of a preference for one of the objects or for the relative location of the novel and familiar objects was controlled by counterbalancing these variables.

These considerations suggest that the post-training anesthetic treatment impaired retention performance of object recognition by affecting memory consolidation, although a proactive effect of anesthesia on retrieval cannot be fully disregarded [32-34]. One possible explanation for the differential effect of this treatment on memory for object recognition, but not for two-way active avoidance, might be related to the brain regions involved in the memory consolidation of these tasks. Two-way active avoidance is an instrumental conditioning, and appears to be related mainly to striatum and amygdaloid complex [21-25, 35]. In contrast, novel object recognition memory depends on the perirhinal cortex and hippocampus [17-20]. In vitro studies have reported that isoflurane can affect $\mathrm{GABA}_{\mathrm{A}}$ and/or NMDA/nonNMDA receptors, enhancing inhibition on hippocampus and amygdala [36, 37], and may disrupt long-term potentiation and long-term depression in CA1 [38]. Also, an effect of anesthesia on hippocampal neurogenesis and differentiation of new neurons has been observed [1, 2], although some works have failed to find evidences of such an effect $[3,39]$. Those discrepancies might be related to the time when neurogenesis is measured after anesthesia. Isoflurane has also been found to regulate hippocampal expression of several proteins related to synaptic plasticity, stress response, detoxification, and cytoskeleton immediately and several days after anesthesia recovery [40], and to decrease acetilcoline [41] and serotonin [42] release in the hippocampus. Jointly, those data suggest that post-training isoflurane might exert higher effects on memory consolidation of tasks depending on hippocampus than of tasks related to implicit memory systems. Two of the experiments that have studied the effect of post-training isoflurane on memory found improvement in retention of one-way active avoidance [8], and improvement of spatial memory in young rats, and disruption of the same task in aged rats [10]. Spatial tasks are hippocampal-dependant [43-45], and one-way active avoidance also seems to be related to the hippocampus, among other brain regions [46, 47]. However, Butterfield et al. [7], also using spatial task, failed to observe any effect of posttraining isoflurane. Similarly, Dutton et al. [4] found no effects of posttraining isoflurane on fear pavlovian conditioning, either to a tone (which is mainly related to the amygdala) or to a context (an hippocampal dependant task), while low isoflurane doses during training disrupted both tasks.

Along with differences in the brain regions involved in memory consolidation, the tasks used in our work are different in many aspects. The main differences are in reinforcement modality (shock vs. novelty) and motivation level. It is plausible to consider that the motivation level for twoway active avoidance task may be stronger than that for object recognition. This would explain why the former task was not affected by the treatment. Another difference between the two tasks relies in the amount of training needed. Two-way active avoidance requires more training than object recognition. Hence, at the end of the training session the level of avoidance responses was far from asymptotic, so that memory of this task at the end of the acquisition session was probably not very strong. In spite of that, memory for this task was not disrupted by the isoflurane procedures. Neither there was a disruption of further learning during the retention test, since the statistical analyses of the evolution of avoidances from the last ten trials in the acquisition session to the first ten trials in the retention session showed a significant increase in avoidances that did not depend on the group.

In addition to isoflurane, some factors associated to the administration procedure of the anesthetic agent might have contributed to the impairment of object recognition memory observed in our experiments. The procedures used in this work are common in brain research experiments and included induction of anesthesia in a chamber and maintenance by supplying isoflurane through a face mask suitable for being fitted to a stereotaxia apparatus. The confinement of the animals to the induction chamber may induce some degree of stress. Much evidence has shown that stress, as well as stress-related hormones, such as epinephrine and glucocorticoids, can modulate memory $[13,15,16,48,49]$. There is extensive evidence that the amygdala is critically involved in mediating this memory modulatory effect and that projections from the amygdala modulate memory consolidation occurring in other brain regions, such as hippocampus [13]. Therefore, stress could have differentially modulated the long-term object recognition memory task, in part depending on hippocampus, without affecting the other task, which is mainly related to striatum. However, it has been observed that stress and stress-related hormones can modulate longterm memory both of the object recognition task [48-52], and of two-way active avoidance [12, 26, 53].

The differential effect on object recognition memory and two-way active avoidance observed in our work can also be explained by an interaction between anesthesia itself, the level of arousal of the task, and the stress produced by the anesthetic administration procedure. These combined factors may have caused a stress event taking place after the training session which may have impaired the task associated to a low level of arousal (i.e., object recognition task in rats habituated previously to the learning context), while sparing the other task associated to a high level of arousal. Concerning the two-way active avoidance task, previous studies have shown that post-training epinephrine $[12,26]$ or induction of a moderate stress [53] can improve long-term 
retention. But in the present experiment this improvement was not observed, maybe because the stress associated to the administration of the anesthetic is relatively low compared to the stress associated with the task itself. In view of the stated data, it is unlikely that stress alone can explain the differential effects found on object recognition and on two-way active avoidance after posttraining isoflurane administration, although stress may have contributed to the isoflurane effect.

To summarise, the global anesthesia procedure with isoflurane immediately after the training session impaired object recognition memory, but did not affect the two-way active avoidance task. We suggest that this differential effect could depend, among other factors, on the level of arousal/ stress of the learning task, and the brain region involved in memory consolidation of these tasks. Our results could have important implications for memory studies that require the administration of post-training treatments under anesthesia, even when the anesthesia is as short as $15 \mathrm{~min}$.

\section{ACKNOWLEDGEMENTS}

P.J. was a recipient of a pre-doctoral grant from the Ministerio de Ciencia y Tecnología. The research was funded by the Ministerio de Ciencia y Tecnología and FEDER (BSO2003-04113/PSCE).

\section{REFERENCES}

[1] Zhu C, Gao J, Karlsson N, et al. Isoflurane anesthesia induced persistent, progressive memory impairment, caused a loss of neural stem cells, and reduced neurogenesis in young, but not adult, rodents. J Cereb Blood Flow Metab 2010; 30: 1017-30.

[2] Stratmann G, Sall JW, May LD, et al. Isoflurane differentially affects neurogenesis and long-term neurocognitive function in 60day-old and 7-day-old rats. Anesthesiology 2009; 110: 834-48.

[3] Stratmann G, Sall JW, Bell JS, et al. Isoflurane does not affect brain cell death, hippocampal neurogenesis, or long-term neurocognitive outcome in aged rats. Anesthesiology 2010; 112: 305-15.

[4] Dutton RC, Maurer AJ, Sonner JM, Fanselow MS, Laster MJ, Eger EI. Isoflurane causes anterograde but not retrograde amnesia for pavlovian fear conditioning. Anesthesiology 2002; 96: 1223-9.

[5] Rammes G, Starker LK, Haseneder R, et al. Isoflurane anaesthesia reversibly improves cognitive function and long-term potentiation (LTP) via an up-regulation in NMDA receptor $2 \mathrm{~B}$ subunit expression. Neuropharmacology 2009; 56: 626-36.

[6] Banerjee U, Das P. Comparative study of retrograde amnesia in rats on active and passive avoidance tasks and spontaneous recovery of memory. Psychopharmacology (Berl) 1977; 53: 299-303.

[7] Butterfield NN, Graf P, Ries CR, MacLeod BA. The effect of repeated isoflurane anesthesia on spatial and psychomotor performance in young and aged mice. Anesth Analg 2004; 98: 1305-11.

[8] Komatsu H, Nogaya J, Anabuki D, et al. Memory facilitation by posttraining exposure to halothane, enflurane, and isoflurane in ddN mice. Anesth Analg 1993; 76: 609-12.

[9] Komatsu H, Nogaya J, Kuratani N, Ueki M, Yokono S, Ogli K. Repetitive post-training exposure to enflurane modifies spatial memory in mice. Anesthesiology 1998; 89(5): 1184-90.

[10] Culley DJ, Baxter M, Yukhananov R, Crosby G. The memory effects of general anesthesia persist for weeks in young and aged rats. Anesth Analg 2003; 96: 1004-9.

[11] Abt JP, Essman WB, Jarvik ME. Ether-induced retrograde amnesia for one-trial conditioning in mice. Science 1961; 133: 1477-8.

[12] Costa-Miserachs D, Portell-Cortes I, Aldavert-Vera L, TorrasGarcia M, Morgado-Bernal I. Long-term memory facilitation in rats by posttraining epinephrine. Behav Neurosci 1994; 108: 46974.
[13] McGaugh JL. The amygdala modulates the consolidation of memories of emotionally arousing experiences. Annu Rev Neurosci 2004; 27: 1-28.

[14] Lupien SJ, Maheu F, Tu M, Fiocco A, Schramek TE. The effects of stress and stress hormones on human cognition: Implications for the field of brain and cognition. Brain Cogn 2007; 65: 209-37.

[15] Sandi C, Pinelo-Nava MT. Stress and memory: Behavioral effects and neurobiological mechanisms. Neural Plast 2007; 78970.

[16] Row BW, Dohanich GP. Post-training administration of corticotropin-releasing hormone $(\mathrm{CRH})$ enhances retention of a spatial memory through a noradrenergic mechanism in male rats. Neurobiol Learn Mem 2008; 89: 370-8.

[17] Broadbent NJ, Squire LR, Clark RE. Spatial memory, recognition memory, and the hippocampus. Proc Natl Acad Sci USA 2004; 101: 14515-20

[18] de Lima MN, Luft T, Roesler R, Schroder N. Temporary inactivation reveals an essential role of the dorsal hippocampus in consolidation of object recognition memory. Neurosci Lett 2006; 405(1-2): 142-6.

[19] Dere E, Huston JP, De Souza Silva MA. The pharmacology, neuroanatomy and neurogenetics of one-trial object recognition in rodents. Neurosci Biobehav Rev 2007; 31: 673-704.

[20] Winters BD, Saksida LM, Bussey TJ. Object recognition memory: Neurobiological mechanisms of encoding, consolidation and retrieval. Neurosci Biobehav Rev 2008; 32: 1055-70.

[21] McDonald RJ, White NM. A triple dissociation of memory systems: Hippocampus, amygdala, and dorsal striatum. Behav Neurosci 1993; 107: 3-22.

[22] Van Golf Racht-Delatour B, El Massioui N. Rule-based learning impairment in rats with lesions to the dorsal striatum. Neurobiol Learn Mem 1999; 72: 47-61

[23] Yin HH, Ostlund SB, Knowlton BJ, Balleine BW. The role of the dorsomedial striatum in instrumental conditioning. Eur J Neurosci 2005; 22: 513-23.

[24] Atallah HE, Lopez-Paniagua D, Rudy JW, O'Reilly RC. Separate neural substrates for skill learning and performance in the ventral and dorsal striatum. Nat Neurosci 2007; 10: 126-31.

[25] Segura-Torres P, Aldavert-Vera L, Gatell-Segura A, RedolarRipoll D, Morgado-Bernal I. Intracranial self-stimulation recovers learning and memory capacity in basolateral amygdala-damaged rats. Neurobiol Learn Mem 2010; 93: 117-26.

[26] Torras-Garcia M, Portell-Cortes I, Costa-Miserachs D, MorgadoBernal I. Long-term memory modulation by posttraining epinephrine in rats: Differential effects depending on the basic learning capacity. Behav Neurosci 1997; 111: 301-8.

[27] Ennaceur A, Michalikova S, Bradford A, Ahmed S. Detailed analysis of the behavior of lister and wistar rats in anxiety, object recognition and object location tasks. Behav Brain Res 2005; 159: 247-66.

[28] Mostafa RM, Mostafa YM, Ennaceur A. Effects of exposure to extremely low-frequency magnetic field of $2 \mathrm{G}$ intensity on memory and corticosterone level in rats. Physiol Behav 2002; 76: 589-95.

[29] Marti-Nicolovius M, Portell-Cortes I, Morgado-Bernal I Improvement of shuttle-box avoidance following post-training treatment in paradoxical sleep deprivation platforms in rats. Physiol Behav 1988; 43: 93-8

[30] Hineline PN. Warmup in avoidance as a function of time since prior training. J Exp Anal Behav 1978; 29: 87-103.

[31] Irifune M, Sato T, Nishikawa T, et al. Hyperlocomotion during recovery from isoflurane anesthesia is associated with increased dopamine turnover in the nucleus accumbens and striatum in mice. Anesthesiology 1997; 86: 464-75.

[32] Crosby C, Culley DJ, Baxter MG, Yukhananov R, Crosby G. Spatial memory performance 2 weeks after general anesthesia in adult rats. Anesth Analg 2005; 101: 1389-92.

[33] Culley DJ, Baxter MG, Crosby CA, Yukhananov R, Crosby G. Impaired acquisition of spatial memory 2 weeks after isoflurane and isoflurane-nitrous oxide anesthesia in aged rats. Anesth Analg 2004; 99: 1393-7.

[34] Culley DJ, Baxter MG, Yukhananov R, Crosby G. Long-term impairment of acquisition of a spatial memory task following isoflurane-nitrous oxide anesthesia in rats. Anesthesiology 2004; 100: 309-14

[35] Savonenko A, Werka T, Nikolaev E, Zielinski K, Kaczmarek L. Complex effects of NMDA receptor antagonist APV in the 
basolateral amygdala on acquisition of two-way avoidance reaction and long-term fear memory. Learn Mem 2003; 10: 293-303.

[36] Wakasugi M, Hirota K, Roth SH, Ito Y. The effects of general anesthetics on excitatory and inhibitory synaptic transmission in area CA1 of the rat hippocampus in vitro. Anesth Analg 1999; 88: 676-80.

[37] Caraiscos VB, Newell JG, You-Ten KE, et al. Selective enhancement of tonic GABAergic inhibition in murine hippocampal neurons by low concentrations of the volatile anesthetic isoflurane. J Neurosci 2004; 24: 8454-8.

[38] Simon W, Hapfelmeier G, Kochs E, Zieglgansberger W, Rammes G. Isoflurane blocks synaptic plasticity in the mouse hippocampus. Anesthesiology 2001; 94: 1058-65.

[39] Tung A, Herrera S, Fornal CA, Jacobs BL. The effect of prolonged anesthesia with isoflurane, propofol, dexmedetomidine, or ketamine on neural cell proliferation in the adult rat. Anesth Analg 2008; 106: 1772-7.

[40] Kalenka A, Gross B, Maurer MH, Thierse HJ, Feldmann RE, Jr. Isoflurane anesthesia elicits protein pattern changes in rat hippocampus. J Neurosurg Anesthesiol 2010: 22: 144-54.

[41] Whittington RA, Virag L. The differential effects of equipotent doses of isoflurane and desflurane on hippocampal acetylcholine levels in young and aged rats. Neurosci Lett 2010; 471(3): 166-70.

[42] Whittington RA, Virag L. Isoflurane decreases extracellular serotonin in the mouse hippocampus. Anesth Analg 2006; 103: 928 .

[43] Eichenbaum H. Hippocampus: Mapping or memory? Curr Biol 2000; 10: R785-7.

[44] Kessels RP, de Haan EH, Kappelle LJ, Postma A. Varieties of human spatial memory: A meta-analysis on the effects of hippocampal lesions. Brain Res Brain Res Rev 2001; 35: 295-303.
[45] Smith DM, Mizumori SJ. Hippocampal place cells, context, and episodic memory. Hippocampus 2006; 16: 716-29.

[46] Alvarez EO, Banzan AM. Ventral hippocampal glutamate receptors in the rat: Possible involvement in learning mechanisms of an active avoidance response. J Neural Transm 1999; 106: 987-1001.

[47] Cimadevilla JM, Fenton AA, Bures J. Functional inactivation of dorsal hippocampus impairs active place avoidance in rats. Neurosci Lett 2000; 285: 53-6.

[48] Okuda S, Roozendaal B, McGaugh JL. Glucocorticoid effects on object recognition memory require training-associated emotional arousal. Proc Natl Acad Sci USA 2004; 101(3): 853-8.

[49] Jurado-Berbel P, Costa-Miserachs D, Torras-Garcia M, CollAndreu M, Portell-Cortes I. Standard object recognition memory and "what" and "where" components: Improvement by posttraining epinephrine in highly habituated rats. Behav Brain Res 2010; 207: 44-50.

[50] Dornelles A, de Lima MN, Grazziotin M, et al. Adrenergic enhancement of consolidation of object recognition memory. Neurobiol Learn Mem 2007; 88: 137-42.

[51] Maroun M, Akirav I. Arousal and stress effects on consolidation and reconsolidation of recognition memory. Neuropsychopharmacology 2008; 33: 394-405.

[52] Roozendaal B, Castello NA, Vedana G, Barsegyan A, McGaugh JL. Noradrenergic activation of the basolateral amygdala modulates consolidation of object recognition memory. Neurobiol Learn Mem 2008; 90: 576-9.

[53] Coll-Andreu M, Marti-Nicolovius M, Morgado-Bernal I. Facilitation of shuttle-box avoidance by the platform method: Temporal effects. Physiol Behav 1991; 49: 1211-5.

(C) Jurado-Berbel et al.; Licensee Bentham Open.

This is an open access article licensed under the terms of the Creative Commons Attribution Non-Commercial License (http://creativecommons.org/licenses/by$\mathrm{nc} / 3.0 /$ ), which permits unrestricted, non-commercial use, distribution and reproduction in any medium, provided the work is properly cited. 